\title{
Analysis of the dynamical behavior of piezoceramic actuators using piezoelectric isogeometric finite elements
}

\author{
Christian Willberg* \\ Institute of Composite Structures and Adaptive Systems, German Aerospace Center (DLR), Lilienthalplatz 7, \\ 38108 Braunschweig, Germany
}

(Received August 13, 2015, Revised November 23, 2015, Accepted November 27, 2015)

\begin{abstract}
In this paper an electromechanically coupled isogeometric finite element is utilized to analyse Lamb wave excitation with piezoceramic actuators. An effective actuator design reduces the energy needed for Lamb wave excitation, which is beneficial if a structural health monitoring system should be applied for a structure. For a better understanding of the actuator behavior the piezoeceramics are studied both free and bonded at a structure. The numerical part of the analysis is performed utilizing isogeometric finite elements. To obtain the optimal performance for the numerical analysis the effect of k-refinement of the isogeometric element with respect to the convergence is studied and discussed. The optimal numerical setup with the best convergence rate is proposed and is validated with free piezoeceramic actuators. The validated model is then utilized to study the impact of actuator shape and adhesive bondline effect to the wave amplitude. The study shows that simplified analytical equations do not predict the optimal excitation frequencies for all piezoceramic designs accurately.
\end{abstract}

Keywords: FEM; isogeometric analysis; piezoelectric; smart structures

\section{Introduction}

Lamb wave based structural health monitoring systems are one promising approach to monitor thin walled structures, e.g., aeroplanes Boller et al. (2009), Giurgiutiu (2008). Lamb waves are ultrasonic waves and therefore interact with damages Mook et al. (2003). They are characterized by a low geometrical attenuation $(1 / \sqrt{ } \mathrm{r}) \mathrm{Su}$ and $\mathrm{Ye}(2009)$. Therefore they are promising for monitoring large areas of light weight CFRP (composite fiber reinforced plastics) structures, e.g., wings of airplanes, because it is possible to monitor great distances or surfaces with a relatively low number of sensors and actuators. However, Lamb waves have complex properties, e.g., they are dispersive, occur in at least two basic modes (symmetric and anti-symmetric) and under special conditions these modes can convert into each other. For a conversion between the symmetric and anti-symmetric mode the wave has to travel through a discontinuity, which is not symmetric to the center plane of the wave and vice versa Ahmad and Gabbert (2012), Willberg et al. (2012b).

Numerous modeling approaches have been developed to describe the complex properties of

*Corresponding author, Ph.D., E-mail: christian.willberg@dlr.de 
Lamb waves efficiently, Willberg et al. (2015). One of these approaches is the so called isogeometric element concept. This concept wants to close the gap between computer aided design (CAD) and finite element analysis (FEA) to reduce the modeling effort Hughes et al. (2005). The reduction of the meshing time and an accurate description of the geometry increase the efficiency of numerical simulations. By assuming accurate so called water-tight geometrical decription from a CAD tool the isogeometric concept utilizes the functional description of the geometry from the CAD software (B-splines, NURBS, T-splines, etc.) and reuses them as shape function for the FEA Cottrell et al. (2009). Assuming that in the near future the application of isogeometric elements will be common, then also special multi-physics finite elements are required, e.g., for the simulation of piezoelectric smart structures. The development of an isogeometric piezoelectrical element has been done by Willberg and Gabbert (2012). It has been shown that this isogeometric element needs a lower number of degrees of freedom due to its exact geometrical description. For a dynamic analysis the performance of a two-dimensional isogeometric finite element has been studied by Willberg et al. (2012a). In this study compared to other higher order approaches isogoemtric finite elements show better convergences. Therefore, a lower number of degrees-offreedom is needed to reach the same accuracy as other higher order element and as result it leads to an increase in memory efficiency. On the contrary the time integration strategy has to be improved because, to the authors knowledge, no method for mass matrix diagonalization for isogeometric elements exists which lead to accurate results for higher frequencies. Adam et al. (2015) analyzes several lumping strategies, but for greater eigenfrequencies the eigenmodes show errors and the accuracy decreases. Although the memory cost is lower the computational time is not reduced compared to spectral finite elements. To understand reason of the better convergence rate of the isogeometric element the effect of the inter-element-continuity is studied. With the effective modeling approach the behavior of the actuator due to Lamb wave excitation is studied. The results are based on the experimental findings of Pohl et al. (2012). The piezoelectrical finite element is validated with experimental data of several unbounded piezoceramic actuators by analyzing the eigenfrequencies and eigenmodes. In the last part a two-dimensional analysis is performed to study the effect of stiffness, geometry and adhesive layer variation.

The paper is structured as following. First, the isogeometric piezoelectric finite element is derived. Second the effect to the convergence due to k-refinement is analysed. Third, the experimental findings of Pohl et al. (2012) are briefly introduced to motivate the further analysis. In the fourth section the paper is concluded.

\section{Theoretical background}

The derivation of the piezomechanical isogeometric finite element has been adopted from Willberg and Gabbert (2012). In this section the material law, the basic finite element equations and the non-uniform rational B-spline (NURBS) representation are introduced. The isogeometric finite element is derived for small deformations and linearized material behavior.

\subsection{Isogeometric piezomechanical model}

Piezoelectric materials are able to transform mechanical deformation in electrical voltage (direct piezoelectric effect) and vice versa (converse piezoelectric effect). To model a piezoelectric elastic material in a low voltage range, the linearized piezoelectric constitutive equations are 
sufficient to describe the coupled electromechanical behavior Ikeda (1996). The linearized piezomechanical material law can be written as

$$
\begin{gathered}
\boldsymbol{\varepsilon}=\mathbf{S}^{E} \boldsymbol{\sigma}+\mathbf{d E}, \\
\mathbf{D}=\mathbf{d}^{T} \boldsymbol{\sigma}+\boldsymbol{e}^{\sigma} \mathbf{E} .
\end{gathered}
$$

The parameters $\boldsymbol{\sigma}, \boldsymbol{\varepsilon}, \mathbf{E}, \mathbf{D}, \mathbf{S}^{E}, \mathbf{d}$ and $\boldsymbol{e}^{\sigma}$ are the mechanical stresses, the mechanical strains, the electric field, the electrical displacement, the elastic compliance matrix measured at constant electric field, the piezoelectric coupling matrix and the permittivity measured at constant mechanical stress, respectively. The mechanical strain is related to the displacement $\mathbf{u}$ as $\boldsymbol{\varepsilon}=\boldsymbol{D} \mathbf{u}$, where $\boldsymbol{D}$ is the differential operator. The electric field is defined as the gradient of the electrical potential $\Phi$ as

$$
\mathbf{E}=-\operatorname{grad} \Phi
$$

Based on this material law the isogeometric finite element formulation can be derived. The difference to the equally formulated standard finite elements is the NURBS shape functions.

\subsection{Isogeometric finite element formulation}

The equations of motion of a piezoelectric continuum can be derived using Hamilton's principle, which states that the motion of the system within the time interval $\left[t_{1}, t_{2}\right]$ is such that the variation of action vanishes, i.e., the motion of the system takes the path of stationary action Marinkovic et al. (2006)

$$
\delta \int_{t_{1}}^{t_{2}}(L+W) d t=0
$$

where

$$
L=\frac{1}{2} \int\left(\rho \dot{\mathbf{u}}^{T} \dot{\mathbf{u}}-\boldsymbol{\varepsilon}^{T} \boldsymbol{\sigma}-\mathbf{E}^{T} \mathbf{D}\right) d V
$$

represents the Lagrangian of the system and includes the kinetic energy as well as the potential mechanical and electrical energies. The external work $W$ is given as

$$
W=\int_{V} \mathbf{u}^{T} \mathbf{F}_{V} d V+\int_{S_{1}} \mathbf{u}^{T} \mathbf{F}_{V} d S_{1}+\sum_{i=1}^{n} \mathbf{u}_{i}^{T} \mathbf{F}_{i}-\int_{S_{2}} \Phi Q_{S_{2}} d S_{2}-\sum_{j=1}^{m} \Phi_{j} Q_{j}
$$

and includes all external loads and external electrical charges. The vectors $\mathbf{F}_{V}, \mathbf{F}_{\mathrm{S} 1}, \mathbf{F}_{\mathrm{i}}$ are given mechanical loads related to a volume $\mathbf{V}$, to a surface $S_{1}$ and to an element node (a control point for an isogeometric element) $i$, respectively. The scalar values $Q_{S 1}$ and $Q_{j}$ are the electric charge related to a surface $S_{2}$ and to an element node (a control point for an isogeometric element) $j$. The vector $\mathbf{u}$ contains the unknown mechanical displacements and $\Phi$ describes the unknown electrical potential. After substituting Eqs. (5) and (6) into Eq. (7) and rearranging the expression we obtain the variational formulation in the form Samal et al. (2005) 


$$
\begin{gathered}
0=-\int_{V}\left[\rho \delta \mathbf{u}^{T} \ddot{\mathbf{u}}+\delta \boldsymbol{\varepsilon}^{T} \mathbf{C}^{E} \boldsymbol{\varepsilon}-\delta \boldsymbol{\varepsilon}^{T} \boldsymbol{e}^{T} \mathbf{E}-\delta \mathbf{E}^{T} \mathbf{e} \boldsymbol{\varepsilon}-\delta \mathbf{E}^{T} \boldsymbol{e}^{\sigma} \mathbf{E}\right] d V \\
+\int_{V} \delta \mathbf{u}^{T} \mathbf{F}_{V} d V+\int_{S_{1}} \delta \mathbf{u}^{T} \mathbf{F}_{S_{1}} d S_{1}+\sum_{i=1}^{n} \delta \mathbf{u}_{i}^{T} \mathbf{F}_{i}-\int_{S_{2}} \delta \Phi^{T} Q_{S_{2}} d S_{1}+\sum_{i=1}^{n} \delta \Phi_{i} Q_{i}
\end{gathered}
$$

where the vector $\ddot{\mathbf{u}}$ is the the acceleration. The material matrices $\mathbf{C}^{E}=\left(\mathbf{S}^{E}\right)^{-1}$ and $\mathbf{e}=\mathbf{C}^{E} \mathbf{d}$ are the elasticity matrix and the matrix of piezoelectric coupling constants, respectively.

When using the finite element approach, a continuous body is discretized in small subdomains. All displacement and electrical potential fields are approximated within each local domain. The displacements $\mathbf{u}$ and the electrical potential $\Phi$ in a local domain (element) can be expressed in terms of the nodal displacements and the nodal electrical potentials $\left(\mathbf{U}_{\text {cont }}, \Phi_{\text {cont }}\right)$ and the matrices of the mechanical and electrical interpolation functions $\left(\mathbf{H}_{u}, \mathbf{H}_{\phi}\right)$ as Zienkiewicz and Taylor (2000)

$$
\mathbf{u}=\mathbf{H}_{u} \mathbf{U}_{\text {cont }} \quad \text { and } \quad \Phi=\mathbf{H}_{\phi} \boldsymbol{\phi}_{\text {cont }}
$$

This formula is similar to the geometrical description of NURBS shown in Eq. (14). In an isogeometric element non-uniform rational B-splines (NURBS) are used as shape functions and the nodal displacements and the nodal electrical potentials correspond to those at the control points.

The deflections and the electrical potentials at any point of the finite element of the structure can be obtained using Eq. (8). Substituting Eq. (8) together with Eq. (1) and Eq. (2) into the variational formulation Eq. (7), results in the discretized form of the equations of motion of a piezoelectric continuum

$$
\begin{gathered}
\mathbf{M}_{u u} \ddot{\mathbf{U}}_{\text {cont }}+\mathbf{K}_{u u} \mathbf{U}_{\text {cont }}+\mathbf{K}_{u \phi} \boldsymbol{\phi}_{\text {cont }}=\mathbf{f}_{\text {ext }} \\
\mathbf{K}_{\phi u} \mathbf{U}_{\text {cont }}-\mathbf{K}_{\phi \phi} \boldsymbol{\Phi}_{\text {cont }}=\mathbf{q}_{\text {ext }}
\end{gathered}
$$

As already mentioned the matrices of the mechanical and electrical interpolation functions $\left(\mathbf{H}_{u}\right.$, $\mathbf{H}_{\phi}$ ) (see Eq. (8)) contain NURBS as shape functions needed to formulated the isogeometric finite element. NURBS have several advantages in describing curved geometries Willberg and Gabbert (2012). They are derivable with a defined non-uniform knot vector $\mathbf{V}$, B-spline basis function $\mathbf{N}_{i, p}$, weights parameters $w_{i}$ and control points $\mathbf{P}_{i}$. A B-spline basis is comprised of piece-wise polynomials joined with prescribed continuity conditions. To define a B-spline of polynomial order $p$ in one dimension one needs to understand the notion of a knot vector. A knot vector is a set of coordinates in a parametric space, written as

$$
\mathbf{V}=\left[\beta_{0}, \beta_{1}, \beta_{2}, \ldots, \beta_{n_{\text {cont }}+p}, \beta_{n_{\text {cont }}+p+1}\right] \quad \text { with } \quad \beta_{i} \leq \beta_{i+1},
$$

where $i$ is the knot index, $i=0,1, \ldots, n_{\text {cont }}+p+1, \beta_{i}$ is the $i^{\text {th }}$ knot and $n_{\text {cont }}$ is the total number of control points Bazilevs (2006). There are various ways to define B-spline basis functions, but for computer implementation the application of a recurrence formula is the most common way de Boor (1972). The first order basis functions $N_{i, 0}(\beta)$ of polynomial degree $p=0$ are

$$
N_{i, 0}(\beta)= \begin{cases}1, & \text { if } \beta \in\left[\beta_{i}, \beta_{i+1}\right) \\ 0, & \text { otherwise. }\end{cases}
$$


The basis functions $N_{i, p}(\beta)$ of higher order $p>0$ are defined as

$$
N_{i, p}(\beta)=\frac{\beta-\beta_{i}}{\beta_{i+p}-\beta_{i}} N_{i, p-1}(\beta)+\frac{\beta_{i+p+1}-\beta}{\beta_{i+p+1}-\beta_{i+1}} N_{i+1, p-1}(\beta),
$$

where the indices $i$ and $p$ denote the $i$ th basis function of polynomial order $p$. Utilizing the B-spline basis functions $N_{i, p}(\beta)$, the NURBS basis function $R_{i}(\beta)$ can be defined as

$$
R_{i}^{p}(\beta)=\frac{N_{i, p}(\beta) w_{i}}{\sum_{j=1}^{n_{\text {cont }}} N_{j, p}(\beta) w_{j}},
$$

where $w_{i}$ is a weight corresponding to the $i$-th B-spline basis function $N_{i, p}$. An arbitrary NURBS curve can be described as Piegl and Tiller (1995)

$$
\mathbf{X}(\beta)=\sum_{i=1}^{n_{\text {cont }}} R_{i}^{p}(\beta) \mathbf{P}_{i}
$$

The vector $\mathbf{X}$ is the position vector of the described curve. $\mathbf{P}_{i}$ are control points in global cartesian coordinates $\left[x_{1}, x_{2}, x_{3}\right]$. A NURBS curve can be interpreted as projection of a B-spline curve from $\mathfrak{R}^{n+1}$ to a defined surface in $\mathfrak{R}^{n}$ Cottrell et al. (2009). NURBS are projective invariant and if the weights are non-negative the curve lays in the convex hull of the control polygon Bazilevs (2006). This projection is controlled by weight parameters $w_{i}$. The derivatives of the NURBS basis functions needed for the finite element formulation are given as Piegl and Tiller (1995)

$$
\frac{d}{d \beta} R_{i}^{p}(\beta)=w_{i} \frac{W(\beta) N_{i, p}^{\prime}(\beta)-W^{\prime}(\beta) N_{i, p}(\beta)}{W(\beta)},
$$

with

$$
W(\beta)=\sum_{j=1}^{n_{\text {cont }}} N_{j, p}(\beta) w_{j}
$$

and

$$
N_{i, p}^{\prime}(\beta)=\frac{p}{\beta_{i+p}-\beta_{i}} N_{i, p-1}(\beta)+\frac{p}{\beta_{i+p+1}-\beta_{i+1}} N_{i+1, p-1}(\beta) .
$$

For a two or three dimensional finite element two or three dimensional NURBS formulation is needed, given by Cottrell et al. (2009).

\section{Influence of the inter-element-continuity to the convergence rate}

In previous studies done by Willberg et al. (2012a) the improved convergence rate of isogeometric finite elements by simulating Lamb waves have been shown.

In this study the $C^{p-1}$-continuous elements have been utilized. In this section the impact of the inter-element-continuity or k-refinement on the convergence rate is investigated. The setup is equal to the previous studies done by Willberg et al. (2012a). The model is given in Fig. 1.

The frequency has been chosen as $f=477.5 \mathrm{kHz}$. In this frequency range (for a plate thickness 


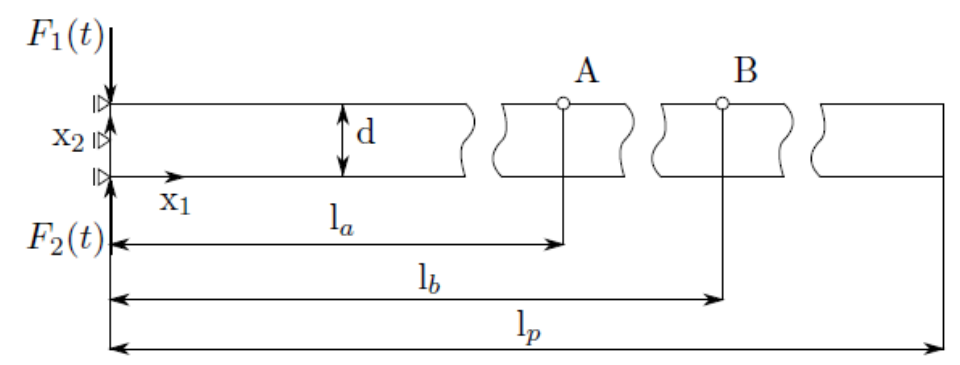

Fig. 1 Two-dimensional model with loads and boundary conditions for the convergence study. Two point forces $F_{1}(t)=\hat{F} \sin (\omega \mathrm{t}) \sin ^{2}(\omega t / 2 n)$ and $F_{2}(t)=a F_{1}(t)$ are applied, with $a=1$ for the excitation of a purely symmetric Lamb wave mode $\left(\mathrm{S}_{0}\right)$ and $a=-1$ if the anti-symmetric mode $\left(\mathrm{A}_{0}\right)$ is considered. The dimensions of the aluminium (see Table 5) plate are : $l_{a}=100 \mathrm{~mm}, l_{b}=200 \mathrm{~mm}$, $l_{p}=500 \mathrm{~mm}, h=2 \mathrm{~mm}$

of $h=2 \mathrm{~mm}$ ) the dispersion, describing the frequency-dependence of both the group and phase velocity is low for the fundamental Lamb wave modes.

To determine the quality of the finite element solution the time-of-flight $t_{c}$ of the propagating Lamb wave package between the points A and B is utilized. To get a defined package position a Hilbert transformation is performed to calculate an envelope at both positions A and B. The centroids of these envelopes are defined as the position of the wave package Willberg et al. (2012a). With this definition the time-of-flight computed using the finite element method ( $t c_{\text {num }}$, type $)$ is compared to the value given by the analytical solution $\left(t c_{a n a}\right)$. Basically using this methodology the comparison of time-of-flights equals the evaluation of resulting group speeds, since dispersive effects are almost excluded due to the narrow bandwidth of the excitation signal and due to the constant distance between the evaluation points. However, the remaining small dispersive effects enter the analytically $\left(t c_{\text {ana }}\right)$ and numerically $\left(t c_{\text {num, type }}\right)$ computed time-of-flight parameters to an equal extent, hence the presented convergence indicator is able to quantify the Lamb wave behaviour even for very low orders of the errors magnitude.

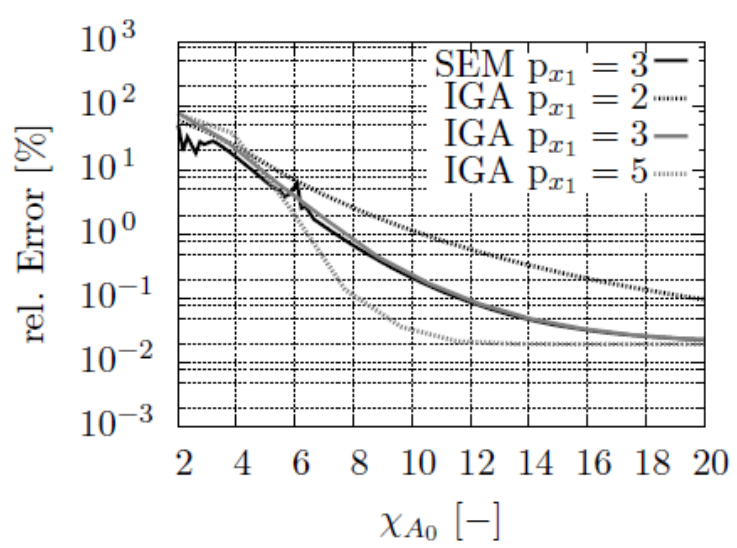

(a) $\mathrm{A}_{0}$-mode

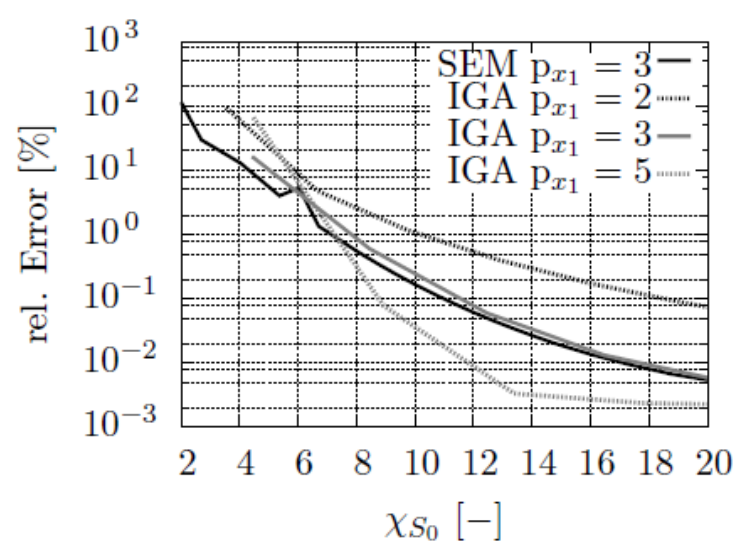

(b) $\mathrm{S}_{0}$-mode

Fig. 2 Convergence curve of isogeometric elements with $C^{1}$-continuity $\left(p_{x 3}=4\right)$ 
For the study of the k-refinement convergence effects, the polynomial order in thickness direction is chosen as $p_{x 3}=4$. If a polynomial degree $p_{x 3}=4$ keep locking to a minimum and greater polynomial degrees does not lead for the chosen frequency to an error reduction Willberg et al. (2012a). Two different inter-element-continuities in $\mathrm{x}_{1}$-direction are chosen; $C^{1}$ - and $C^{0}$-continuity. The $C^{0}$-continuity corresponds to the standard finite element inter-element-continuity, meaning that no jumps in the displacement functions occur.

Fig. 2 shows the result of the convergence test for the $C^{1}$-continuous isogeometric finite elements. As reference the convergence curve of the spectral finite elements (SEM) with the optimal polynomial degree $p x_{1}=3$ (minimal memory effort to reach a $1 \%$ error for the time of flight) is plotted Willberg et al. (2012a). These elements have been chosen because they are commonly used for higher order Lamb wave simulation. Due to their formulation a lumped mass matrix is obtained which has advantages for the time-integration Duczek (2014). The convergence rate for the $\mathrm{A}_{0^{-}}$as well as the $\mathrm{S}_{0}$-mode are lower in comparison to $C^{p-1}$-continuous isogeometric elements, compare with Willberg et al. (2012a), cf. Fig. 4. They are steady and have no peaks. The convergence of the isogeometric elements of order $p x_{1}=3$ are almost equal to the reference curve of SEM of the same order. Due to the difference in polynomial order as expected the accuracy of the isogeometric element in comparison to the SEM elements is for $p x_{1}=2$ and for the polynomial degree $p x_{1}=5$ the accuracy is higher compared to the SEM elements. For an equal polynomial order $\left(p x_{1}=3\right)$ the accuracy of the isogeometric finite elements are slightly lower compared to the SEM, but the maximum accuracy is equal for both formulations.

Fig. 3 illustrates the results of the $C^{0}$-continuous isogeometric elements. The curves are steady except the peak for $p x_{1}=3$ in the $\mathrm{A}_{0}$-mode curve. This behavior can be attributed to local element eigenfrequencies and correlate to the peak in the SEM curve Schmicker et al. (2014). Again the convergence rate of order $p x_{1}=3$ of the $\mathrm{A}_{0^{-}}$and $\mathrm{S}_{0^{-}}$-mode is almost similar to the reference SEM convergence, but the accuracy of the isogeometric finite elements is lower until they reach the maximum accuracy.

Generally, isogeometric finite elements with a lower inter-element-continuity exhibit worsened convergence compared to a $C^{p-1}$-continuous element. Also the element eigenfrequency effects described by Schmicker et al. (2014) occur. The maximum achievable accuracy of lower interelement-continuity elements is not improved and equal to $C^{p-1}$-continuous elements. Fig. 4

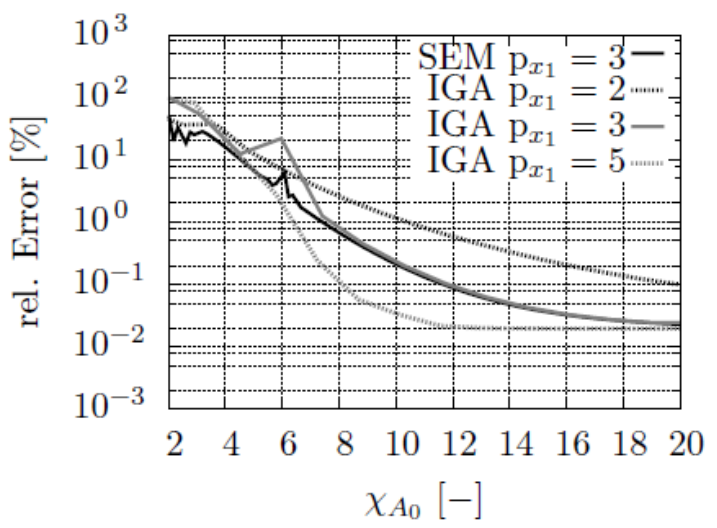

(a) $\mathrm{A}_{0}$-mode

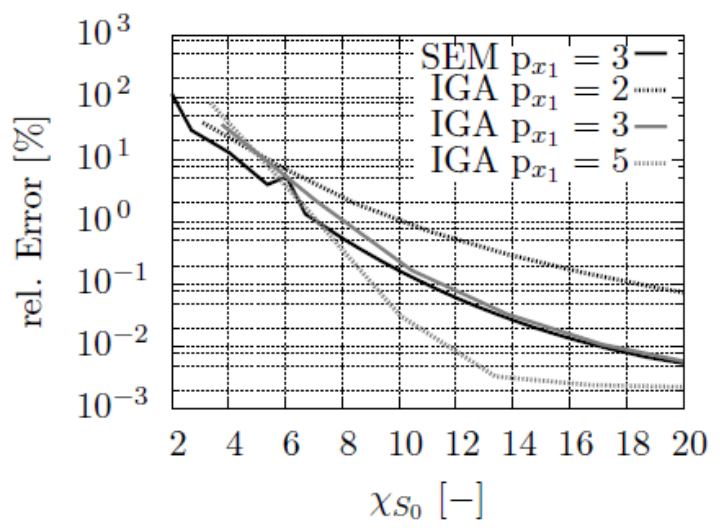

(b) $\mathrm{S}_{0}$-mode

Fig. 3 Convergence curve of isogeometric elements with $C^{0}$-continuity $\left(p_{x 3}=4\right)$ 


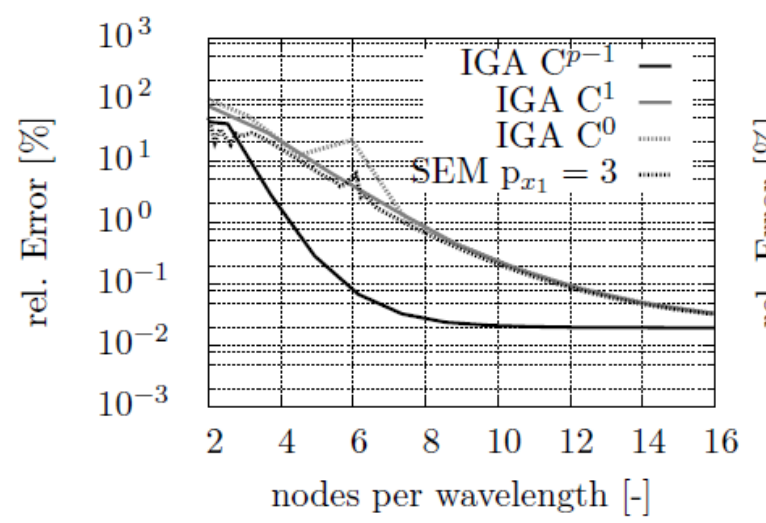

(a) $\mathrm{A}_{0}$-mode

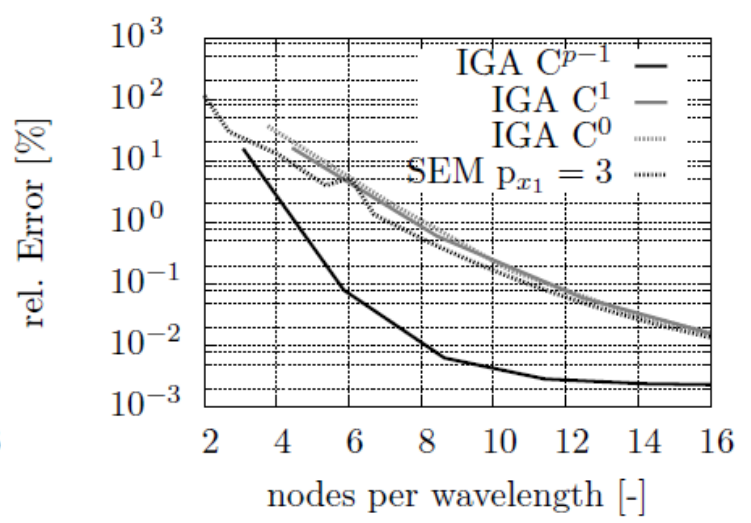

(b) $\mathrm{S}_{0}$-mode

Fig. 4 Convergence curve of isogeometric elements with inter-element- different continuity $\left(p_{x 1}=3, p_{x 3}=4\right)$

illustrates this conclusion for a polynomial degree of $p x_{1}=3$. For $C^{1}$ and $C^{0}$ inter-element-continuity no improvement in convergence is reached in comparison to the spectral finite element solution of equal order.

Therefore, $C^{p-1}$-continuous isogeometric finite elements are to be preferred when dealing with Lamb wave propagation. This statement agrees with Evans et al. (2009) for a theoretical approximation analysis which said that a numerical comparison of the classical finite element and $k$-refinement methods revealed that the $k$-method has better approximation properties than the classical finite element method on a per degree-of-freedom basis, further suggesting that the $k$ refinement method is an accurate and robust scheme for approximating solutions to partial differential equations. These results are consistent with observations made previously based on discrete Fourier analysis and the numerical solutions of boundary value problems.

\section{Actuator behavior}

The vibration of a free-free piezoceramic discs is measured and compared with a threedimensional isogeometric finite element model solution. The resonance frequencies as well as the modeshapes (visually) are compared. This analysis is used to validate the three-dimensional IGA model. The general mode shapes are the same for the coupled and free disc case. The analysis is motivated by the excitation of Lamb waves. Therefore, a frequency range $10 \mathrm{kHz} \leq f \leq 300 \mathrm{kHz}$ is chosen, because for $2 \mathrm{~mm}$ plate thicknesses no higher order Lamb wave modes occur.

The eigenmodes of a general circular plate can be separated in different types. Tangential modes, which are described by Huang et al. (2004) are excluded here since they have a weak appearance. Also the thickness modes are not considered. For piezoceramic plates with thicknesses less than $2 \mathrm{~mm}$ considered here they occur at frequencies higher than $1 \mathrm{MHz}$ PIC. These frequencies are outside the investigated frequency domain $10 \mathrm{kHz} \leq f \leq 300 \mathrm{kHz}$. Only bending and radial modes are studied in this investigation. If the piezoceramic is applied to a structure these types of eigenmodes will primarily activate Lamb waves. Moreover, both mode types exist in the regarded frequency domain where only the two basic Lamb wave modes arise. Fig. 5(a) displays the used coordinate system and the direction of the polarization of the piezoceramic discs of 

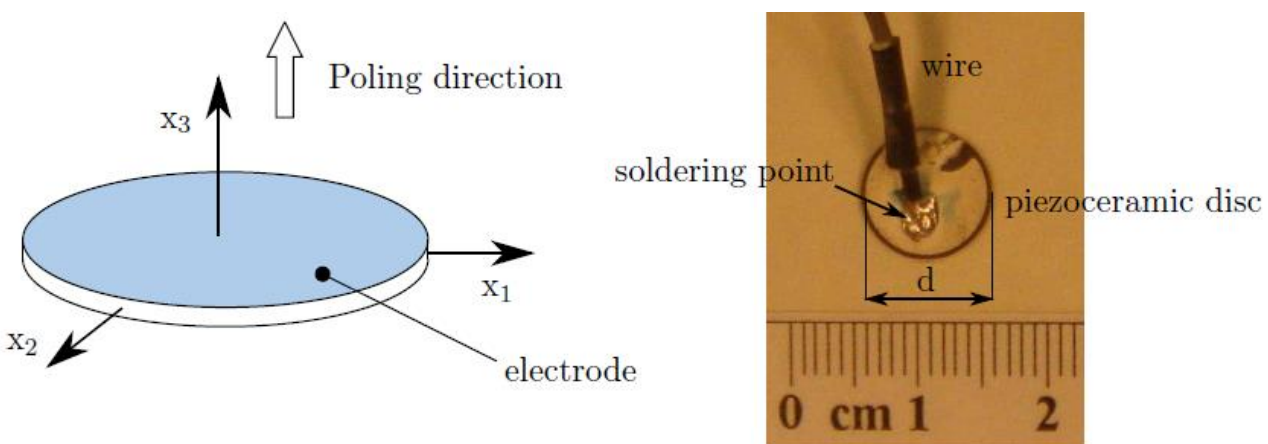

(a) Coordinates of the piezoceramic disc of (b) Example for a real piezoceramic disc $(d=10$ diameter $d$ and thickness $h$ $\mathrm{mm}, h=0.5 \mathrm{~mm}$, PIC-181)

Fig. 5 Uncoupled piezoceramic disc

Table 1 Comparison of measurement (M.) of free piezoceramics with the isogeometric solution (S.) (1 - PIC$151, d=10 \mathrm{~mm}, h=1 \mathrm{~mm} ; 2$ - PIC-181, $d=10 \mathrm{~mm}, h=0.5 \mathrm{~mm} ; 3$ - PIC-181, $d=10 \mathrm{~mm}, h=2 \mathrm{~mm} ; 5$ - PIC-181, $d=16 \mathrm{~mm}, h=1 \mathrm{~mm} ; 6$ - PIC-181, $d=16 \mathrm{~mm}, h=2 \mathrm{~mm} ; 7$ - PIC-151, $d=40 \mathrm{~mm}, h=0.5 \mathrm{~mm}$ )

\begin{tabular}{|c|c|c|c|c|c|c|c|c|c|}
\hline & S. & M. & $1[\mathrm{kHz}]$ & $2[\mathrm{kHz}]$ & $3[\mathrm{kHz}]$ & $4[\mathrm{kHz}]$ & $5[\mathrm{kHz}]$ & $6[\mathrm{kHz}]$ & $7[\mathrm{kHz}]$ \\
\hline M. & & & 28.9 & 17.7 & 38.8 & 59.7 & 12.9 & 23.4 & - \\
\hline S. & & & 27.3 & 16.7 & 32.4 & 58.6 & 12.97 & 24.7 & 0.8 \\
\hline M. & & & 58.1 & 33.2 & 63.1 & 109.5 & 24.3 & 48.1 & 1.9 \\
\hline S. & & & 53 & 32.2 & 61.4 & 106.1 & 24.9 & 46.4 & 1.77 \\
\hline M. & & & 63.5 & 40.1 & 74.4 & 123.3 & 29 & 55.3 & 2.05 \\
\hline S. & & & 60.9 & 38.6 & 72.1 & 120.7 & 29.7 & 54.1 & 2.1 \\
\hline M. & & & 106.8 & 68.1 & 124 & 192.1 & 49.4 & 90.4 & 3.7 \\
\hline S. & & & 101.8 & 66.9 & 120.6 & 187.9 & 51.1 & 88.6 & 3.7 \\
\hline M. & & & 117.5 & 72.5 & 132.2 & 199.6 & 53.2 & 95.8 & 4.15 \\
\hline S. & & & 107.3 & 69.6 & 125.2 & 191.3 & 53.4 & 91.4 & 3.9 \\
\hline M. & & & 156.6 & 103.5 & 179.7 & 263.5 & 74.6 & 129.8 & 5.5 \\
\hline S. & & & 147.8 & 101 & 175 & 257.3 & 76.4 & 126.1 & 5.7 \\
\hline M. & & & 192.1 & 119.6 & 205.5 & 295.8 & 86.5 & 149 & 6.9 \\
\hline S. & & & 168.2 & 115.2 & 197 & 276 & 86.9 & 140.7 & 6.6 \\
\hline M. & & & 204.5 & 128.7 & 222 & - & 95.2 & 161.3 & 7.6 \\
\hline S. & & & 192.9 & 126.8 & 215 & 301.8 & 95.5 & 153.7 & 7.3 \\
\hline
\end{tabular}

diameter $d$ and thickness $h$. To measure the eigenfrequencies a linear chirp signal is generated utilizing the piezoceramic actuator. The piezoceramic disc is mounted on foam (free) and the 
response of the structure is measured at the bottom surface. Fig. 5(b) shows an example of a free disc made of PIC-181 with the wire and the soldering points at the top surface and a diameter $d=10$ $\mathrm{mm}$ and a thickness $h=0.5 \mathrm{~mm}$. The numerical model neglect soldering points and only the pure circular disc comparable with Fig. 5(a) is modeled.

The eigenfrequencies $f=\omega / 2 \pi$ utilizing the isogeometric finite elements are calculated as

$$
\left(\mathbf{K}_{u u}+\mathbf{K}_{u \phi} \mathbf{K}_{\phi \phi} \mathbf{K}_{\phi u}-\omega_{0}^{2} \mathbf{M}_{u u}\right) \widehat{\mathbf{U}}=0
$$

$\hat{\mathbf{U}}$ is the eigenvector, $\mathbf{K}$ is the stiffness matrix and $\mathbf{M}$ is the mass matrix. The indices $u u, u \phi$, $\phi u$ and $\phi \phi$ denote displacement-displacement, displacement-electrical potential, electrical potential-displacement and electrical potential-electrical potential, respectively (see Eq. (9a) and Eq. (9b)).

\subsection{Bending modes of the piezoelectric circular plate}

Seven piezoceramic discs are studied with different material properties as well as different geometries. The material properties of PIC-151 and PIC-181 are given in Table 6. The out-of-plane component of the velocity of the bottom surface of each disc is measured after applying the external broadband signal. Therefore, no wire or soldering point disturbs the laser beam. Table 1 shows seven measured bending modes compared with an isogeometric finite element solution. The dominant displacements are in $x_{3}$-direction. It must be noted that the wire and soldering points which are illustrated in Fig. 5(b) have only a low influence on the eigendynamic of the discs Willberg et al. (2009). Therefore, they are not included in the numerical model. Nevertheless, the results in Table 1 show a good agreement (error is smaller than $10 \%$ with respect to the numerical solution with exception of one result) between the simulations (S.) and the measurements (M.). The sequence of the mode appearance as well as the mode shapes is similar.

\subsection{Radial modes of the piezoelectric circular plate}

For the comparison of the radial modes with the experimental and the isogeometric results an analytical solution has been used. The radial eigenfrequencies $f_{j}$ correspond to eigenvalues of the characteristic equation of the analytical solutions. The eigenvalues are only dependent of the Poisson's ratio of the $\mathrm{x}_{1}-\mathrm{x}_{2}$-plane $v_{12}$. For a Poison's ratio of $v_{12}=0.34$ the first four eigenvalues $z_{j}$ are given by Giurgiutiu (2008) as

$$
z_{1}=2.074 ; \quad z_{2}=5.397 ; \quad z_{3}=8.576 ; \quad z_{4}=11.73
$$

With the values of $z_{j}$ the eigenfrequencies $f_{j}$ can be directly calculated as

$$
f_{j}=\sqrt{\frac{1}{S_{11}^{E} \rho\left(1-v_{12}^{2}\right)}} \frac{z_{j}}{\pi d_{\max }}
$$

The experimental identification of the radial modes has been done using 3D scanning laser vibrometry measurements. An example is shown in Fig. 6. All three spectra and the corresponding in-plane mode shape are plotted. The graphs show that the in-plane modes also produce remarkably high secondary out-of-plane components. It has been observed that even in out-ofplane spectra measured with a 1D vibrometer radial mode peaks are to be observed (cf. Fig. 6(c)). 


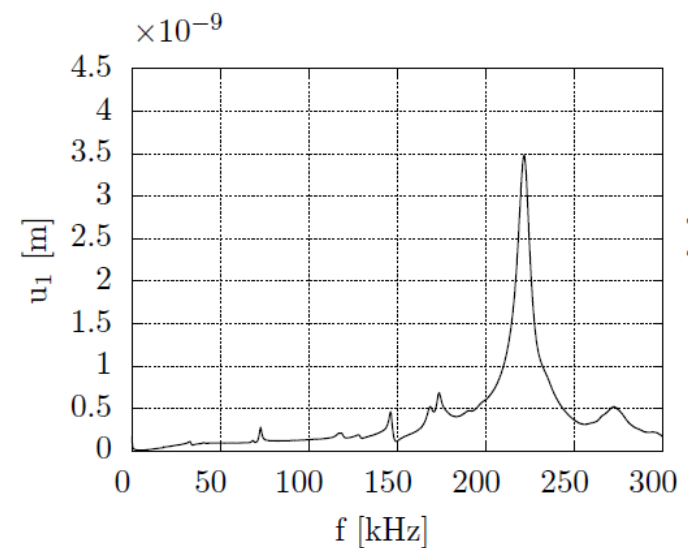

(a) $u_{1}$-displacement

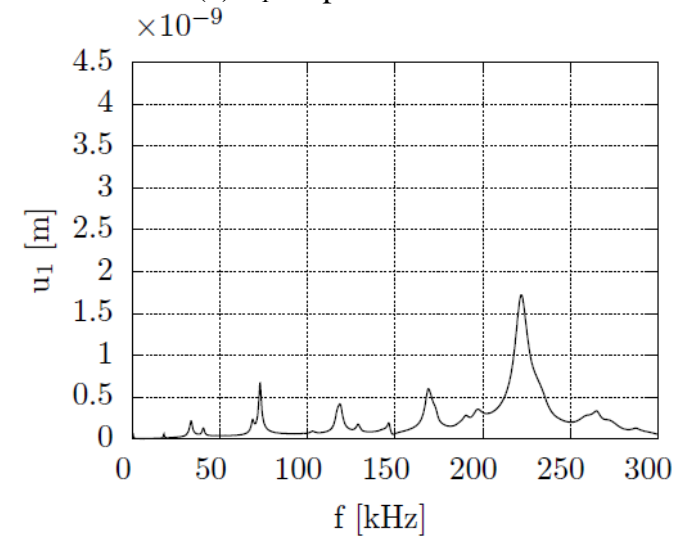

(c) $u_{3}$-displacement

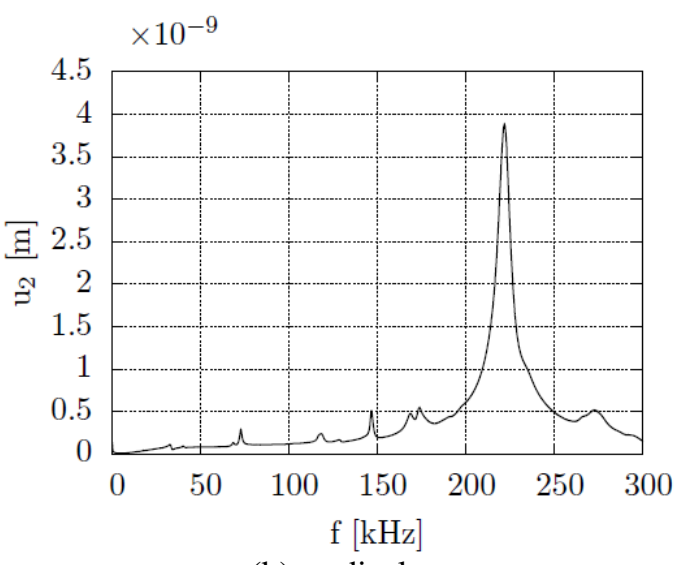

(b) $u_{2}$-displacement

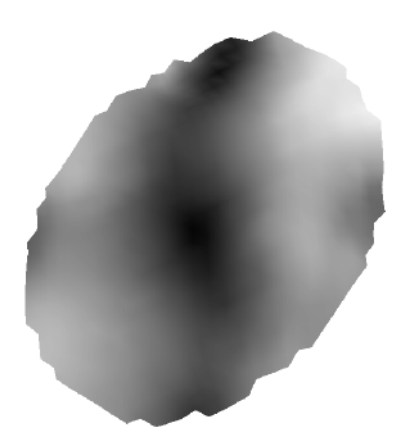

(d) Measured mode shape $u_{\text {mag }}=\sqrt{u_{1}^{2}+u_{2}^{2}}$; $f=222 \mathrm{kHz}$

Fig. $63 \mathrm{D}$ measurement of a piezoceramic $(d=10 \mathrm{~mm}, h=0.5 \mathrm{~mm}$, PIC-181)

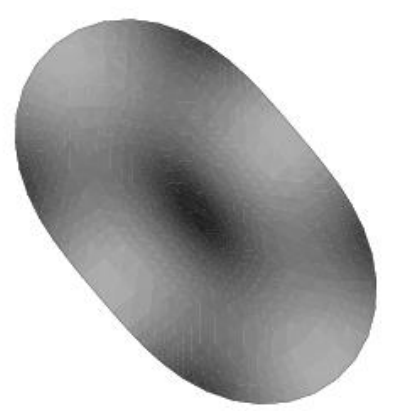

(a) Radial mode 1

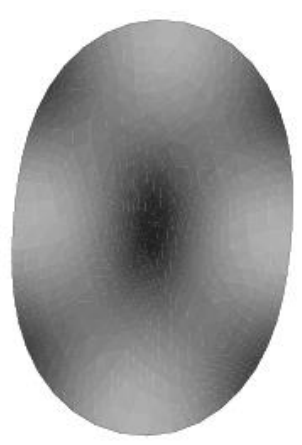

(b) Radial mode 2

Fig. 7 First in-plane eigenmode of the analytical solution Table 2 of an undisturbed piezoceramic

In those cases it is not possible to correlate a fundamental bending mode shape shown in Table 1 (and higher ones too) to the measured peaks. The isogeometric simulations show that many 
bending modes coexist around the higher resonance frequencies of the first radial mode. This is also validated by the experimental results. A splitting of the mode types by 1D (out-of-plane) measurements is not possible. If the in-plane components are observed, the mode shape of an inplane mode can be seen (cf. Fig. 6(d)). Fig. 7 shows the eigenform of the eigenfrequencies plotted in Table 2. Due to ideal symmetry for the numerical or analytical model two modes with the same frequency exists, cf. Fig. 7.

Because, knowing the in-plane spectra are sometimes not enough to identify the radial eigenfrequencies without a doubt, additional measurements have been done. Following Huang et al. (2004) only in-plane and thickness modes cause resonance peaks in the spectrum of electrical impedance. The resonance values obtained by the impedance measurements are given by Pohl et al. (2012). The analytical as well as the isogeometric finite element solution determine all first radial modes correctly. Moreover, as Eq. (19) states, a change of the height of the piezoceramic disc has no influence on the resonance frequencies. Both, the experiments and the isogeometric finite element results show such behavior.

\subsection{Vibration of the coupled piezoceramic actuator}

The design of a piezoceramic patch actuator for structural health monitoring applications should guarantee a most effective Lamb wave generation. According to Kessler et al. (2002) and $\mathrm{Su}$ et al. (2006) the most effective generation of Lamb waves is accomplished, if the actuator length or diameter $d$, respectively, is related to the wave length in the following manner

$$
d=\lambda(n+0.5) \text {. }
$$

$\lambda$ describes the wavelength of the regarded Lamb wave mode and $n$ is an arbitrary integer $n=0,1$, $2, \ldots)$. To investigate the resonance effects a CFRP plate with a $\left[(0 / 90)_{-} f /+45 /-45 /(0 / 90)_{f}\right]_{\text {sym }}$ layup

Table 2 Comparison of the experimental data (3D vibrometry and impedance measurements) with the analytic solution of the radial eigenfrequencies

\begin{tabular}{ccccccc}
\hline $\begin{array}{c}\text { Diameter } \\
{[\mathrm{mm}]}\end{array}$ & Material & $\begin{array}{c}\text { Height } \\
{[\mathrm{mm}]}\end{array}$ & $\begin{array}{c}\text { Analytical } \\
{[\mathrm{kHz}]}\end{array}$ & $\begin{array}{c}\text { Numerical } \\
{[\mathrm{kHz}]}\end{array}$ & $\begin{array}{c}\text { 3D vibrometer } \\
{[\mathrm{kHz}]}\end{array}$ & $\begin{array}{c}\text { Impedance } \\
{[\mathrm{kHz}]}\end{array}$ \\
\hline 10 & PIC-151 & 1 & 190.77 & 192.9 & 195 & 195 \\
40 & PIC-151 & 0.5 & 47.69 & 48.4 & - & 49 \\
10 & PIC-181 & 0.5 & 228.32 & 233.7 & 222 & 225 \\
10 & PIC-181 & 1 & 228.32 & 233 & 225 & 224 \\
10 & PIC-181 & 2 & 228.32 & 230.3 & 224 & 223 \\
\hline
\end{tabular}

Table 3 Optimal frequencies for Lamb mode generation in a CFRP plate up to $500 \mathrm{kHz}$ in $0^{\circ}$-direction Willberg et al. (2013)

\begin{tabular}{cccc}
\hline \hline$n$ & $\lambda[\mathrm{m}]$ & $f$ of $\mathrm{A}_{0}[\mathrm{kHz}]$ & $f$ of $\mathrm{S}_{0}[\mathrm{kHz}]$ \\
\hline 0 & 0.0200 & 40.7 & 276 \\
1 & 0.0067 & 186 & $>500$ \\
2 & 0.0040 & 335 & $>500$ \\
3 & 0.0029 & 480 & $>500$ \\
\hline
\end{tabular}


and a plate thickness of $2 \mathrm{~mm}$ is examined. Table 3 shows the first four optimal wavelengths for this CFRP plate. The values are determined using Eq. (20) in combination with the measured dispersion curves to get the frequencies which are corresponded to the wavelengths $\lambda$ Pohl et al. (2012). The experimental investigation has been done between 0 and $500 \mathrm{kHz}$. Only one optimal excitation frequency of the $\mathrm{S}_{0}$-mode and three for the $\mathrm{A}_{0}$-mode are within the considered frequency range.

Circular shaped piezoceramic actuators with three different heights $(0.5 \mathrm{~mm}, 1 \mathrm{~mm}, 2 \mathrm{~mm})$ and a diameter $d=10 \mathrm{~mm}$ are glued on the CFRP plate. A linear swept-frequency cosine signal is used to excite Lamb waves with multiple frequencies. Two measurements are performed for each piezoceramic. First the surface of the ceramic is scanned to gain the frequency response of the ceramic. The second measurement measures the CFRP plate. The measurement has been done avoiding reflections from the edges. By taking the wave numbers of the $\mathrm{A}_{0^{-}}$and $\mathrm{S}_{0^{-}}$mode it ispossible to plot the frequency response spectra of each mode. Therefore, a 3D FFT is performed on the data of the measured C-scan of the laser vibrometer to detect the temporal periodicities (frequencies) and the spatial periodicities (wave numbers) of the propagating signals Alleyne and Cawley (1991). In doing so the separated frequency amplitude curve can be determined.

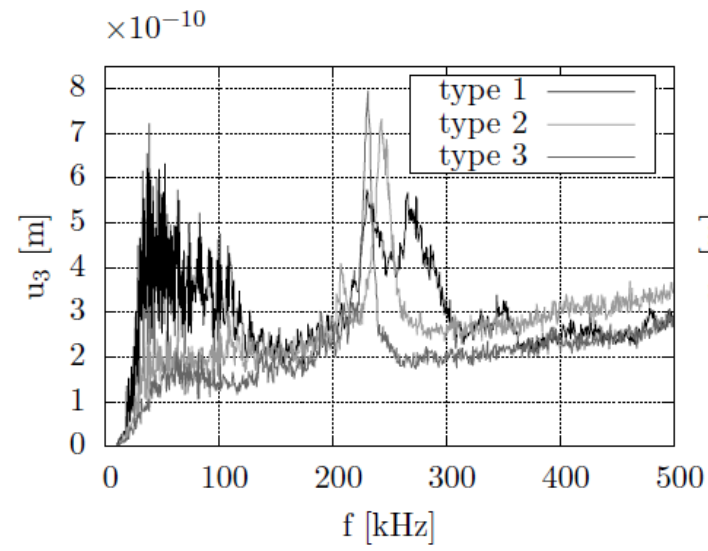

(a) $\mathrm{S}_{0}$-mode spectra

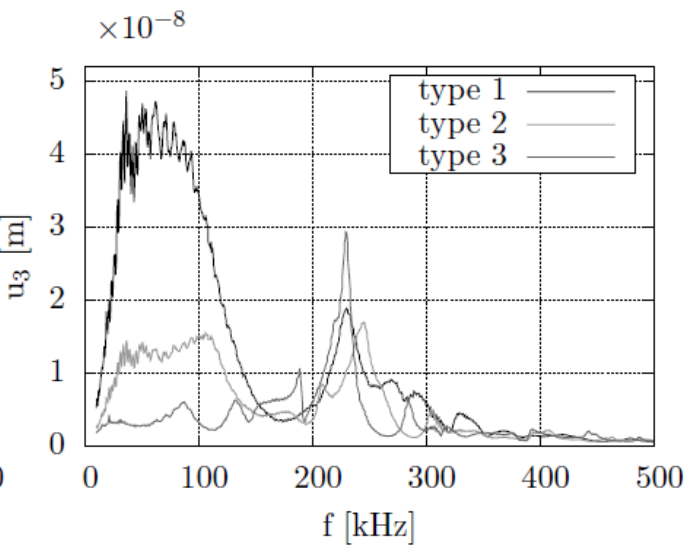

(b) $\mathrm{A}_{0}$-mode spectra

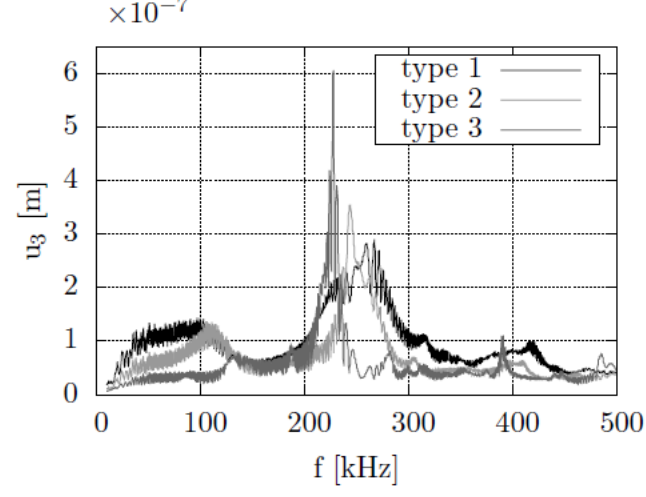

(c) Spectra of piezoceramic disc

Fig. 8 Spectra for the Lamb wave modes for a CFRP plate and different piezocermic discs - Type $1(d=10$ $\mathrm{mm}, h=0.5 \mathrm{~mm})$, Type $2(d=10 \mathrm{~mm}, h=1 \mathrm{~mm})$ and Type $3(d=10 \mathrm{~mm}, h=2 \mathrm{~mm})$ 


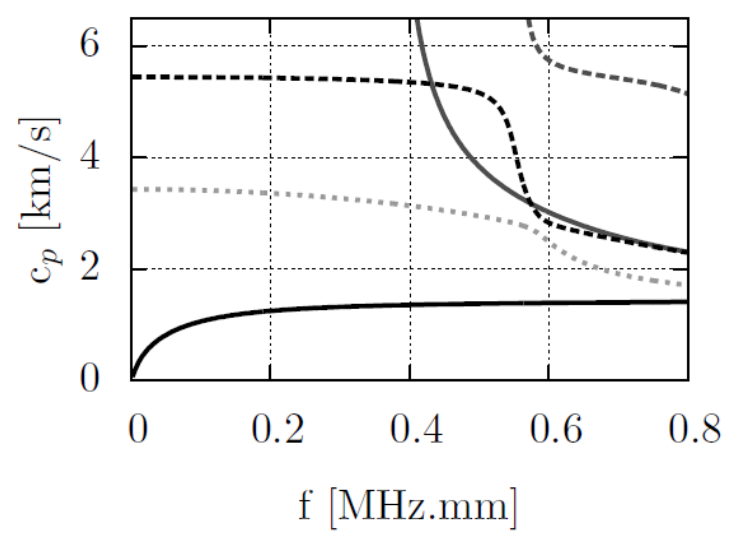

(a) Phase velocity

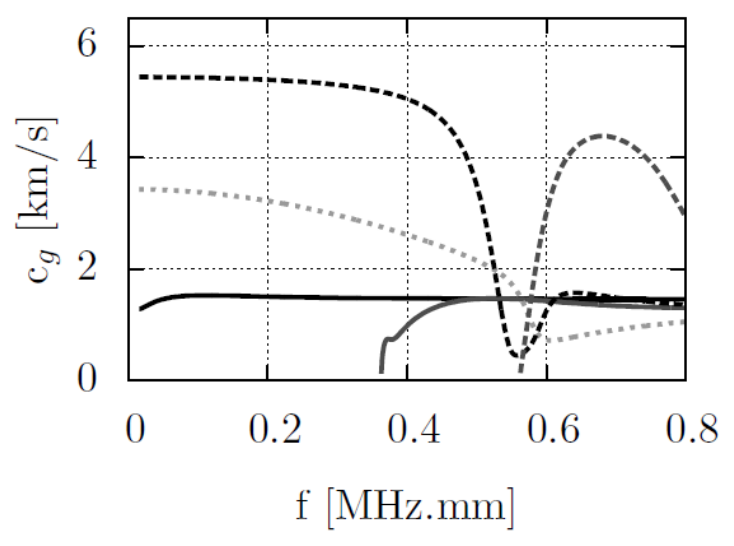

(b) Group velocity

Fig. 9 Dispersion curves of CFRP plate with a $\left[(0 / 90) \_f /+45 /-45 /(0 / 90)_{f}\right]_{\text {sym }}$ layup Ahmad (2011)

If Eq. (20) holds the $\mathrm{A}_{0}$-mode experiences a first maximum at $40.7 \mathrm{kHz}$ and the $\mathrm{S}_{0}$-mode at 276 kHz. The experimental results are displayed in Fig. 8(a)-8(c). The noise in the $\mathrm{S}_{0}$-mode spectra are caused by the great wavelength and the small displacement in $x_{3}$-direction. The different curves from Fig. 8 show a close relation between the resonances of the piezoelectric actuators and the spectra of the Lamb wave modes. The frequencies corresponding to certain values of Lamb wave wavelength for the CFRP plate have been derived from Eq. (20) and are given in Table 3 for frequencies up to $500 \mathrm{kHz}$ both for the $\mathrm{S}_{0^{-}}$and $\mathrm{A}_{0^{-}}$-mode, cf. Fig. 9 for the corresponding dispersion curves. Comparing the measured resonance frequencies (Fig. 8) with the calculated data from Table 3 it can be concluded that Eq. (20) cannot be applied for thicker piezoceramic actuators. For example for a frequency of $f=335 \mathrm{kHz}$ an optimal excitation for the $\mathrm{A}_{0}$-mode should occur. Only the type 1 piezoceramic shows a maximum. It is evident that the eigenfrequencies of the actuator in connection with the structure have a major impact on the excited Lamb wave amplitudes. The assumption that an optimal excitation of Lamb modes occurs if the diameter of the piezoceramic disk is one half of an integer number of the wavelengths does not hold under any circumstance Pohl et al. (2012). Only for thin ceramics it can be confirmed that the lowest eigenfrequency calculated with Eq. (20) coincides with the measurements. In all other cases no agreement with measurements could be observed, meaning that the application of Eq. (20) to estimate optimal excitation frequencies cannot be recommended.

For SHM systems the application of higher frequencies are preferable due to an improved resolution and a better ability to detect damages. The resonances play a major role in the excitation of Lamb waves. For thicker piezoceramics it is the dominant effect which influences the amplitudes of the excited Lamb waves Ha (2010), Mohamed et al. (2011). This effect can be used to reduce the input energy of the actuators. The adhesive layer as mentioned before shifts the position of the resonances. Therefore, a tuning of the optimal frequency should be applied to receive an optimal excitation.

\subsection{Influence parameters to the Lamb wave excitation}

The experiments have shown that the resonances of the coupled piezoceramic actuator have a 


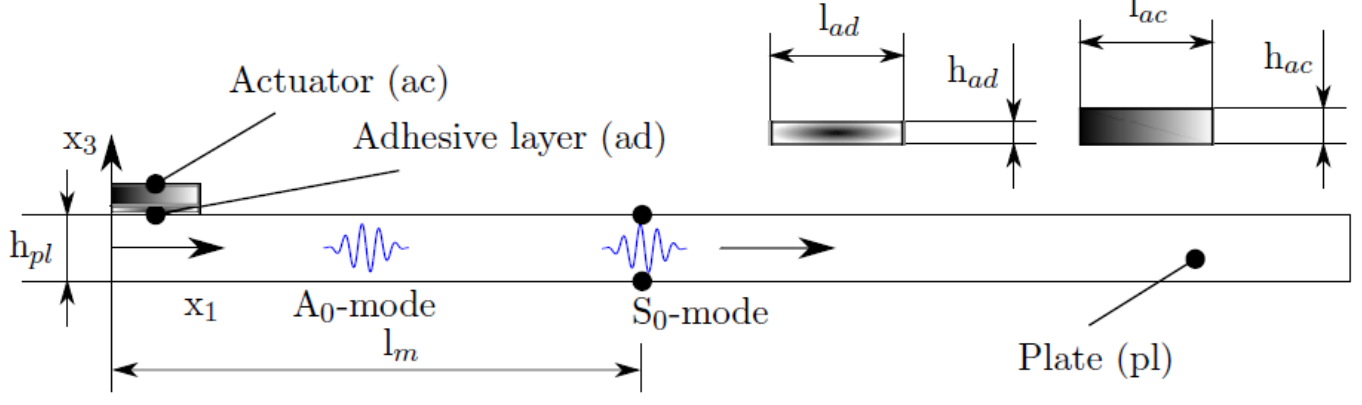

Fig. 10 Two-dimensional symmetric model, used to investigate the influence of the bonding layer to the Lamb wave excitation (actuator: PIC-181; adhesive layer: paraffin wax; plate: aluminum $Y=7 \cdot 10^{10} \mathrm{~N} / \mathrm{m}^{2}$, $v=0.33 \$, l_{p l}=0.6 \mathrm{~m}, h_{p l}=2 \mathrm{~mm}$ ); the results are being measured at a point located at a distance of $l_{m}=50 \mathrm{~mm}$ from the actuator

dominant influence on the excited Lamb waves. In this section the parameters which influence the resonance of the actuator-adhesive layer-host structure system are studied. To investigate the parameters a two-dimensional isogeometric finite element model is created (see Fig. 10). The findings presented in section 3 are used and the optimal discretization scheme is applied to reduce the numerical effort and to gain a good accuracy Willberg et al. (2012a).

Several parameters are varied to study their influence on the resonance of the actuator and the $\mathrm{A}_{0}$ - and $\mathrm{S}_{0}$-mode spectra. The parameters are:

- the thickness of the adhesive layer,

- the Young's modulus of the adhesive layer,

- the thickness of the actuator,

- the length of the actuator and

- the Young's modulus of the plate.

The geometry of the plate remains the same for all simulations. The non-varied properties of the model are given in Table 4. In all given models the rest of the parameters are chosen as in the Table 4.

At the left side of the model (cf. Fig. 10) symmetric boundary conditions $\left(u_{1}\left(x_{1}=0, x_{2}\right)=0\right)$ are applied to reduce the numerical effort. The actuator is made of the piezoelectric material PIC-181 (see Table 6), the plate consists of aluminum (see Table 5) and the adhesive layer is made of paraffin wax (see Table 7). The model is defined by the plate thickness $h_{p l}$, the plate length $l_{p l}$, the actuator thickness $h_{a c}$, the actuator length $l_{a c}$, the adhesive layer thickness $h_{a d}$ and the adhesive layer length $l_{a d}$.

At the top surface of the piezoceramic actuator a constant electrical potential is applied. At the

Table 4 Properties of the model in Fig. 10

\begin{tabular}{cccc}
\hline \hline Parameter & Length $[\mathrm{mm}]$ & Height $[\mathrm{mm}]$ & Material \\
\hline$p l$ & 600 & 2 & Aluminium (Table 5) \\
$a c$ & 5 & 1 & PIC-181 (Table 6) \\
$a d$ & 5 & 0.05 & paraffin wax (Table 7) \\
\hline
\end{tabular}


bottom surface of the actuator the electrical potential is set to zero. A broad-band excitation signal (linear chirp)

$$
\phi(t)=\hat{\phi} \sin \left[2 \pi\left(f_{0}+\frac{f_{1}-f_{0}}{t_{1}} t\right) t\right]
$$

is used. The start frequency $f_{0}$ and end frequency $f_{1}$ are chosen as $f_{0}=10 \mathrm{kHz}$ and $f_{1}=500 \mathrm{kHz}$. The time is given as $t_{1}=1 / f_{0}$.

The displacements of the top surface of the piezoceramic actuator and the displacements of a top $\left(x_{3}=+h_{p l} / 2\right)$ and a bottom $\left(x_{3}=-h_{p l} / 2\right)$ node of the plate in a distance $l_{m}=50 \mathrm{~mm}$ are monitored. The average displacement of the top surface of the piezoceramic is used to determine the spectrum of the applied actuator. The displacements of the top and bottom node of the plate are used to separate the two Lamb modes from each other without performing a 2D FFT. After applying a FFT to the time-dependent data, three spectra are gained similar to Fig. 8.

Fig. 11(a)-Fig. 11(c) show the simulated $u_{3}$-displacement spectra of the two-dimensional model of the two basic Lamb wave modes and the piezoceramic actuator. The model is defined as illustrated in Fig. 10 and Table 4 without varied parameter. As illustrated in the experimental investigation in the previous section there is a dominant coupling between the actuator eigendynamic and the excited Lamb waves. As in the experiments the $\mathrm{A}_{0}$-mode is strongly influenced by the low frequency peaks in the piezoceramic spectrum. This is an indicator for the existence of bending modes in this frequency domain. As shown in the previous section these eigenmodes have a greater influence on the $A_{0}$-mode than on the $S_{0}$ one. On the other side, the first in-plane eigenmode (radial mode for circular shaped actuators in 3D) at $f=237 \mathrm{kHz}$ has a strong impact to the symmetric $\mathrm{S}_{0}$-mode which leads to a high peak in the spectrum on the symmetric mode.

All of the described effects occur (occurrence of in-plane and bending modes of piezoceramic actuators) in each cutting plane perpendicular to the surface of the plate. Therefore, it can be said that the general properties of the coupled actuator-structure system in comparison of the numerical and experimental results can be described by a simple two-dimensional model.

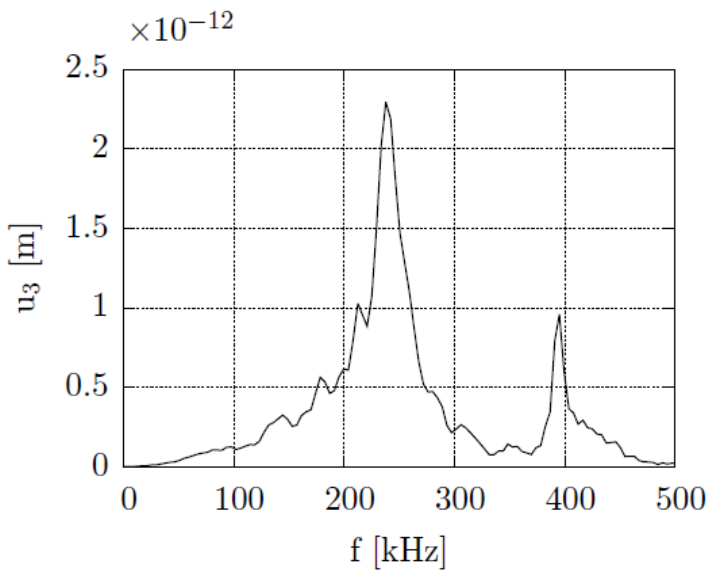

(a) $\mathrm{S}_{0}$-mode spectrum

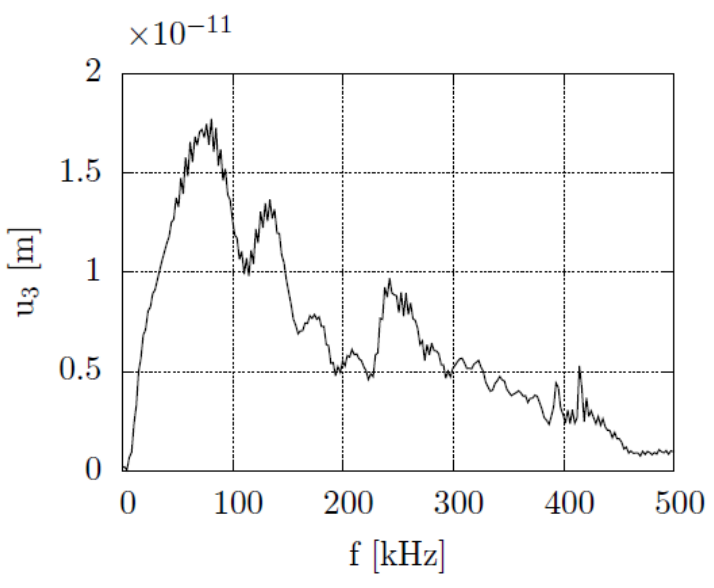

(b) $\mathrm{A}_{0}$-mode spectrum

Fig. 11 Spectra of $u_{3}$-displacement of the two-dimensional model without parameter variation 


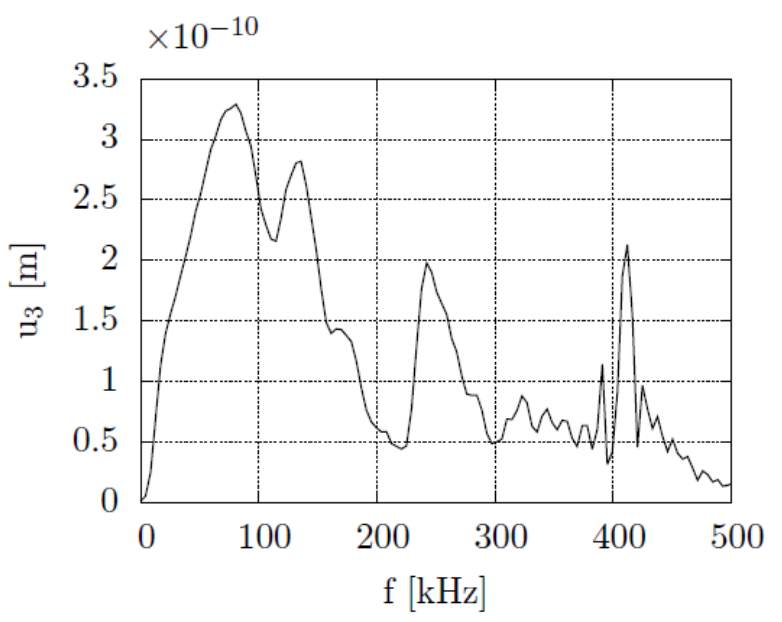

(c) Spectrum of piezoceramic disc

Fig. 11 Spectra of $u_{3}$-displacement of the two-dimensional model without parameter variation

\subsubsection{Influence of the adhesive layer}

The impact of the adhesive layer to the coupling between an actuator and a structure is well known for thin piezoceramic patches. If a patch actuator is glued to the surface of a structure the so called "shear lag" effect can be observed. The "shear lag" is caused by an adhesive layer of finite thickness between the actuator and the host structure (see Fig. 12). The excitation signal from the actuator is transmitted to the structure through interfacial shear stresses within the bonding layer for the most part Sohn and Lee (2010). The "shear lag" causes a reduction of shear strain transfer between the PZT actuator and the host structure. With a lower shear modulus and a thicker adhesive layer, the "shear lag" effect becomes more pronounced. As a result, the signal amplitude is reduced significantly if the resonance effects play a minor role Ha (2009). Due to this effect the effective length of a piezoelectric actuator is smaller than the actual length. Consequently, it can be assumed, that only a fraction of the actual surface of a piezoelectric actuator contributes to the strain transfer. Thus, correction factors have to be applied in order to account for the "shear lag" effect. Based on analytical models (e.g., Euler-Bernoulli beam theory)

Correction factors for various problems assuming the application of thin piezoceramics $(\approx 0.2$ $\mathrm{mm}$ ) only have been derived in Crawley and de Luis (1987), Giurgiutiu (2008), Sirohi and Chopra (2000).

For thicker piezoceramics the resonances of the coupled actuator-structure system play a more important role and superimpose the "shear lag" effect. Moreover, the assumptions made by the Euler-Bernoulli beam theory are not fulfilled in the case of thick ceramics and the correction

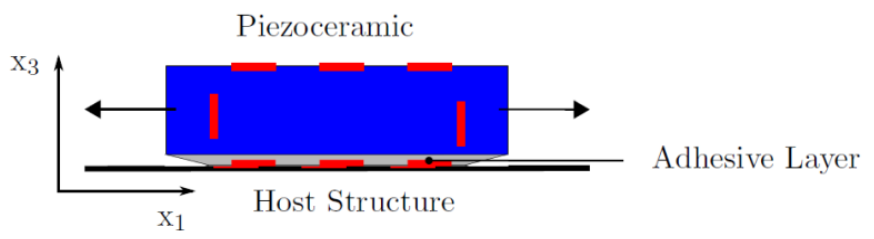

Fig. 12 Schematic representation of the "shear lag" effect 
factors can be overestimated. Therefore, two parameters of the adhesive layer are varied to study their influence to the first resonance of the $\mathrm{S}_{0}$-mode.

The $\mathrm{A}_{0}$-mode spectrum has no clear first resonance peak caused by the first in-plane mode of the piezoceramic. This mode is influenced by the bending modes as well as the in-plane modes. Because, both modes are affected differently by a parameter variation the analysis has been shown that the equal resonance peak of the $\mathrm{A}_{0}$-mode is harder to find for the variations compared to the $\mathrm{S}_{0}$-mode spectra, cf. Fig. 11(a). Therefore, the movement of the resonance peak in the $\mathrm{A}_{0}$-mode spectrum caused by the in-plane mode is hard to determine and no analysis of this spectrum has been done.

Adhesive layer thickness: The adhesive layer thickness $h_{a d}$ is varied between $10 \mu \mathrm{m} \ldots 150 \mu \mathrm{m}$. The change in the first resonance frequency as well as the amplitude of this excitation frequency is observed for the $\mathrm{S}_{0}$-mode. Fig. 13(a) shows the frequency of the first peak in the $\mathrm{S}_{0}$-mode spectrum as a function of the adhesive layer height. The frequency decreases for a greater adhesive layer thickness. As illustrated before, for the experimental model in Fig. 8 and the numerical model in Fig. 11, there is a connection between the dynamic behavior of the piezoceramic actuator and the spectra of the two Lamb wave modes. Because the coupled actuatorstructure system becomes softer the resonance frequencies of the actuator shift to a lower frequencies.

The amplitude of the resonance peak increases (see Fig. 13(b)). This disagrees with the "shear lag" effect, which states that higher thicknesses lead to a reduction in the amplitudes of the excited Lamb wave modes. The amplitude curve is not steady because near the first in-plane eigenfrequency other peaks occur. The in-plane mode is superposed by bending modes. Both mode types are influenced differently by the adhesive layer height and depending on how similar the resonance frequencies of both mode types are, the amplitudes increase or decrease. However, only great changes $(20 \mu \mathrm{m}$ or more) in the adhesive layer thickness lead to a noticeable change in frequency and amplitude. Therefore, the adhesive layer thickness could be used in the design process to alter the eigenfrequencies of the piezoceramic actuator. Small variations $(<10 \%$ thickness variation) caused by the production process are negligible from the original design.

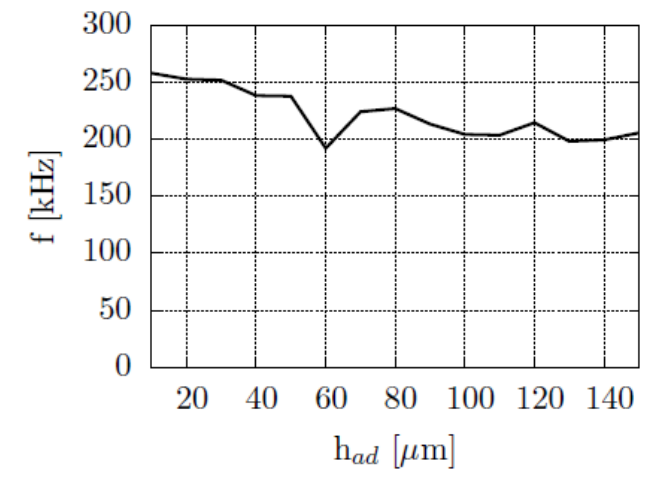

(a) Frequency of the first resonance peak

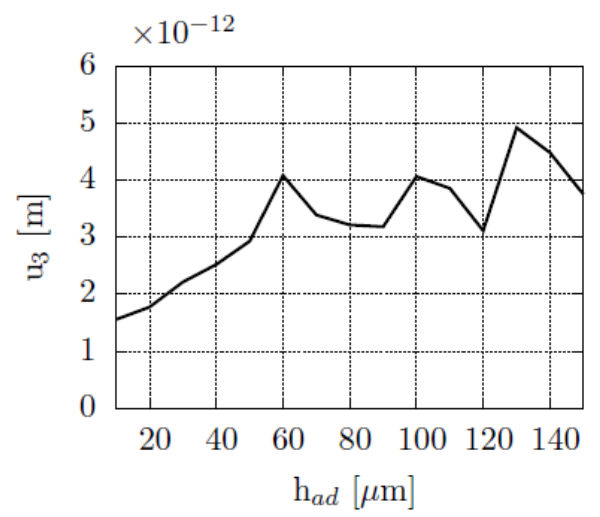

(b) Amplitude of the first resonance peak

Fig. 13 Influence of the thickness $h_{a d}$ of the adhesive layer to the frequency and to the amplitude of the $\mathrm{S}_{0}$-mode 


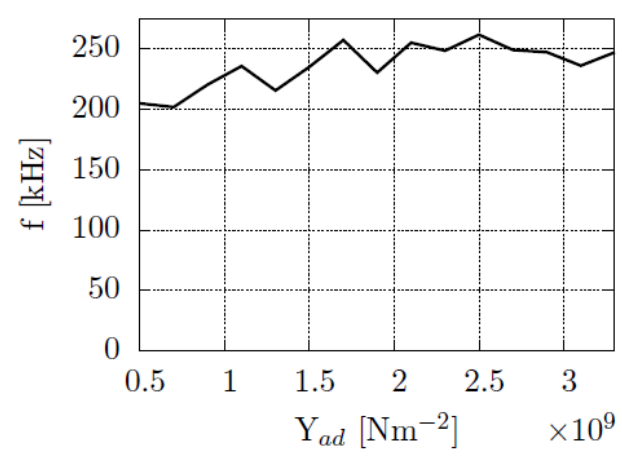

(a) Frequency of the first resonance peak

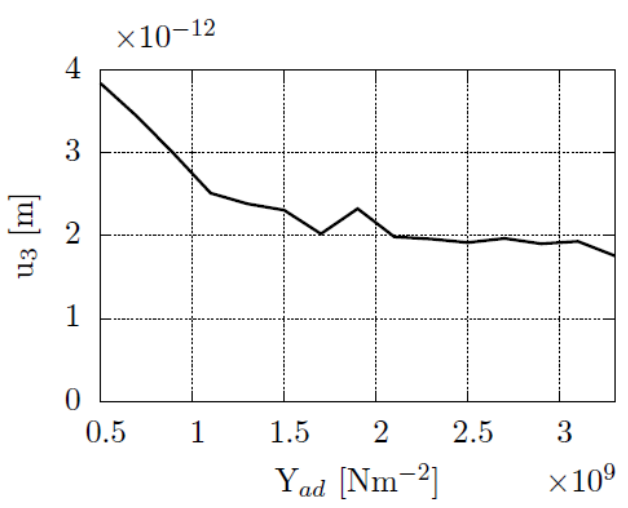

(b) Amplitude of the first resonance peak

Fig. 14 Influence of the Young's modulus $Y_{a d}$ of the adhesive layer to the amplitude of the $\mathrm{S}_{0}$-mode

Adhesive layer stiffness: The adhesive layers Young's modulus $Y_{a d}$ is varied between $0.5 \cdot 10^{9}$ $\mathrm{N} / \mathrm{m}^{2} \ldots 3300 \cdot 10^{9} \mathrm{~N} / \mathrm{m}^{2}$. The lower bound correlates to $50 \%$ of the Young's modulus of paraffin and the upper bound of the Young's modulus correlates to epoxy, which is typically used to glue piezoceramics irreversible to structures. The influence of the stiffness change in the adhesive layer to the frequency of the first peak in the $S_{0}$-mode spectrum is illustrated in Fig. 14(a). For higher Young's moduli the first resonance peak shifts to higher frequencies. The results show that the Young's modulus of different adhesive layers (epoxy or paraffin) does not change the frequency of the first peak drastically.

Fig. 14(b) shows the amplitude of the first peak in the $\mathrm{S}_{0}$-mode spectrum as a function of the Young's modulus of the adhesive layer. Higher Young's moduli cause a reduction in amplitude. This behavior disagrees with the "shear lag" effect and underline that for thicker piezoceramics the eigendynamic superimposes the "shear lag" effect Ha (2010), Willberg et al. (2013). Variations of the material properties of the adhesive layer do not seem to influence the first resonance frequency drastically. Therefore, changes in the material properties due to temperature variations and/or variations caused by a variance in the production process are negligible in the design process.

In summary one can say that the "shear lag" correction factor based on the assumption of the Euler-Bernoulli beam theory does not work correctly in resonance regions of the actuator to predict the amplitude losses caused by adhesive layer changes. Therefore, more detailed analyses are needed.

\subsubsection{Influence of the actuator geometry}

After studying the influence of the adhesive layer on the Lamb wave excitation the impact of the actuator geometry is observed. Firstly, the length is studied and secondly the thickness of the actuator.

Actuator length: The piezoceramic actuator length $l_{a c}$ is varied between $2 \mathrm{~mm} \ldots 16 \mathrm{~mm}$. The influence of this variation on the first resonance frequency is illustrated in Fig. 15(a). There is approximately a $1 / d$ correlation (analytical) between the length of the actuator and the first peak in the $\mathrm{S}_{0}$-mode spectrum caused by the first radial eigenfrequency of the piezoceramic actuator. This coincides with the behavior of the radial eigenfrequencies of the a free circular disc in Eq. (19). 


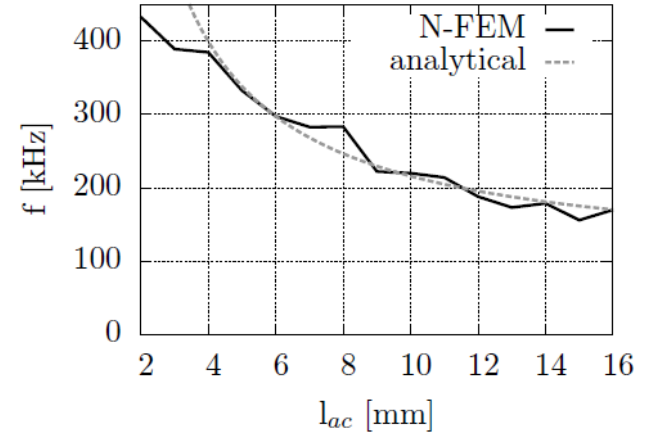

(a) Frequency of the first resonance peak

(a) Frequency of the first resonance peak

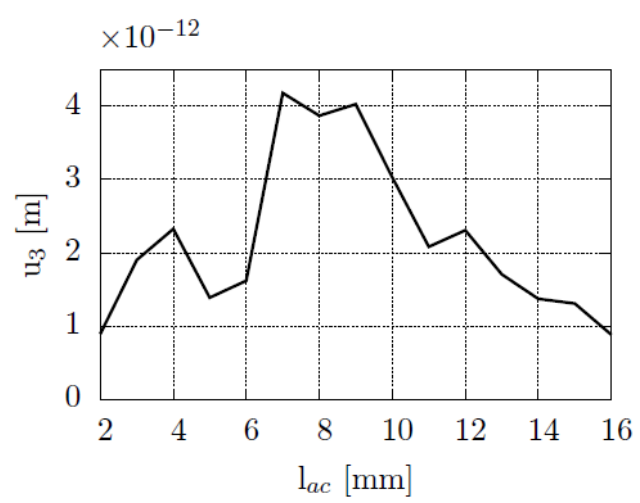

(b) Amplitude of the first resonance peak

(b) Amplitude of the first resonance peak

Fig. 15 Influence of the length $l_{a c}$ of the adhesive layer to the amplitude of the $\mathrm{S}_{0}$-mode

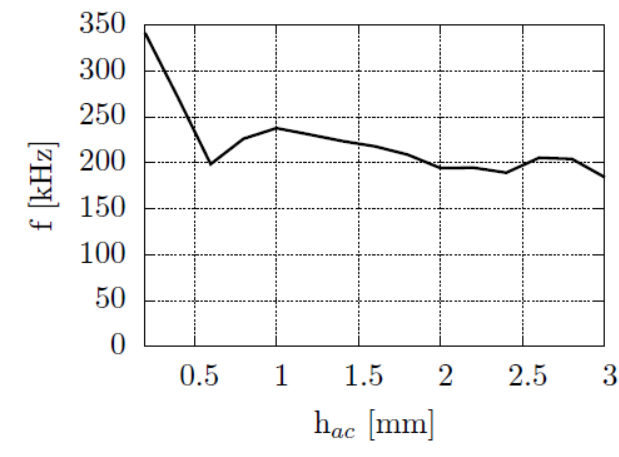

(a) Frequency of the first resonance peak

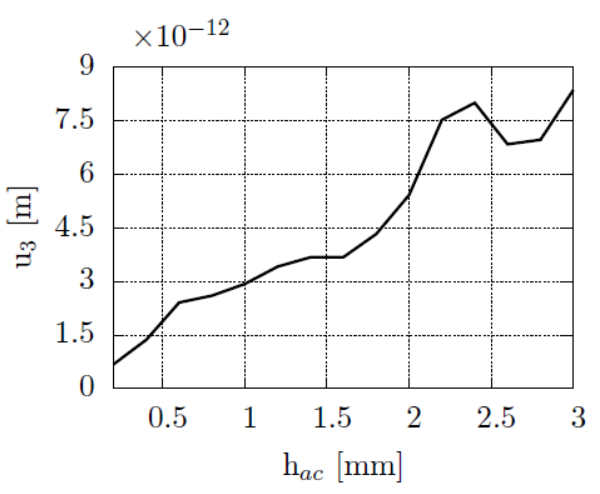

(b) Amplitude of the first resonance peak

Fig. 16 Influence of the thickness $h_{a c}$ of the adhesive layer to the amplitude of the $\mathrm{S}_{0}$-mode

The value of the $u_{3}$-displacement illustrated in Fig. 15(b) first increases and for lengths $l_{a c}>9 \mathrm{~mm}$ decreases.

This behavior could be explained as a result of superposition of the first radial and a bending mode. Both modes are differently influenced by extending the length of the actuator; $1 / d$ for the radial mode and $1 / d^{2}$ for the bending modes Pohl et al. (2012). For lengths between $7 \mathrm{~mm}$ and 10 $\mathrm{mm}$ the frequency of a lower order bending mode correlates with the first radial mode. The optimal diameter calculated with Eq. (20) optimal diameter for the actuator does not agree with the results. That is to say, the changes in the actuator length strongly influence the dynamic behavior. But also here small changes due to the production process can be neglected (variation $<10 \%$ ).

\section{Actuator thickness:}

The piezoceramic actuator thickness $h_{a c}$ is varied between $0.1 \mathrm{~mm} . .3 \mathrm{~mm}$. In Fig. 16(a) the frequency as a function of the actuator thickness is plotted. Changes in the thickness has a low influence the resonance frequency (compared to a change of the length) of the radial eigenmode as 


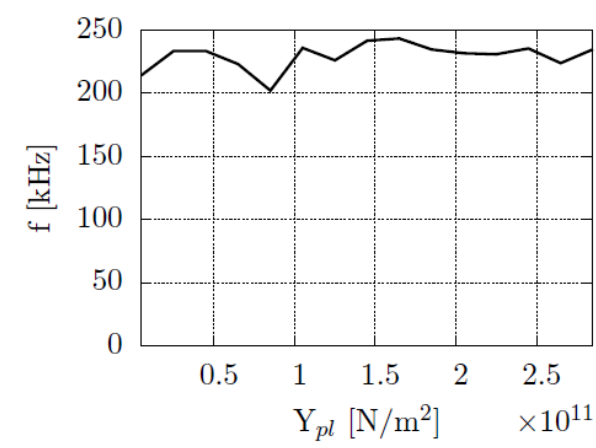

(a) Frequency of the first resonance peak

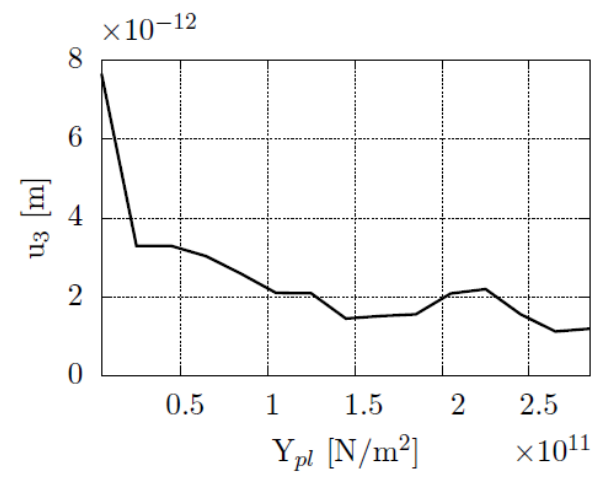

(b) Amplitude of the first resonance peak

Fig. 17 Influence of the Young's modulus $Y_{p l}$ of the plate to the amplitude of the $\mathrm{S}_{0}$-mode

Eq. (19) stated for the free elastic disc, except the strong decrease between $0.2 \mathrm{~mm}$ and $0.6 \mathrm{~mm}$. Experimental and numerical investigations of the free piezoceramic disc show that the eigenfrequencies for thicker piezoceramics are higher. In this research the frequency of the first peak of the $\mathrm{S}_{0}$-mode spectrum is considered. If a bending mode moves near a radial mode, both modes superpose each other and lead to higher amplitudes. The first peak is shifted to lower frequency regions.

The amplitudes of the peaks in the $S_{0}$-mode spectrum are plotted in Fig. 16(b). For thicker piezoceramics the amplitudes increase. The $\mathrm{S}_{0}$-mode is dominantly excited by shear stresses. Therefore, a higher bending stiffness due to the greater actuator thickness leads to a better shear stress transfer into the structure. Moreover, the bending modes are influenced by the greater bending stiffness. The frequency of the lower order bending modes shifts to higher frequencies. The superposed resonances lead then to higher amplitudes as one can see in Fig. 16(b).

\subsubsection{Influence of the plate stiffness}

In this section the influence of the plate stiffness is investigated. The Young's modulus of the plate $Y_{p l}$ is varied between $5 \cdot 10^{9} \ldots 2.1 \cdot 10^{11} \mathrm{~N} / \mathrm{m}^{2}$. The density is constant and chosen as $\rho_{a l u}=2700$ $\mathrm{kg} / \mathrm{m}^{3}$. Fig. 17(a) shows the frequency of the first peak of the $\mathrm{S}_{0}$-mode spectrum as a function of the variation of the Young's modulus. The frequency changes between $200 \mathrm{kHz}$ and $244 \mathrm{kHz}$ without any noticeable connection to the change of the plates Young's modulus. The amplitudes behavior of the first peak of the $\mathrm{S}_{0}$-mode is plotted in Fig. 17(b). For very low a Young's modulus the amplitude is high. In that case the resistance to a deformation of the plate is lower and thus higher amplitudes are possible. In contrast to the frequency behavior in Fig. 17(a) there is a coupling between the amplitude reduction and the plate stiffness, except for a small variation between $Y_{p l}=2 \ldots 2.5 \cdot 10^{11} \mathrm{~N} / \mathrm{m}^{2}$.

\section{Conclusions}

The paper presented the analysis of dynamic effects of piezoceramic actuators due to Lamb wave excitation. To perform this analysis in an effective way isogeometric finite elements have been utilized. It has been shown that a k-refinement causes the better convergence of the elements 
compared to an equal order spectral finite element. Therefore, the conclusion is to use the highest possible continuity to reduce the numerical effort.

The analysis of the dynamic actuator effects have shown, that for coupled piezoceramics the optimal excitation assumption stated by Kessler et al. (2002) is not valid for thicker piezoceramics. To obtain an optimal actuator design a numerical study has been performed. Various parameters have been varied to take the effect of geometry and adhesive bondline into account. The variation of the adhesive layer thickness shows that only great thickness changes influence the position of the first peak in the $\mathrm{S}_{0}$-mode spectrum noticeably. Therefore, this parameter could be used to adapt the position of the first peak. However, small variation in the adhesive layer thickness barely influences the position of the peak and thickness variations during the production process do not have to be taken into account for the design. The Young's modulus of the adhesive layer has a low impact on the first peak in the $\mathrm{S}_{0}$-mode spectrum as well as to the corresponding amplitude. Small variations of the material properties of the adhesive layer during the production process or caused by temperature variations do not change the dynamical behavior of the piezoceramic actuator significantly. The variation of the actuator length shows that for longer patches the frequency of the first peak in the $\mathrm{S}_{0}$-mode spectrum decreases. This correlates with the analytical formulation of the radial eigenfrequency of a circular disc. The corresponding amplitudes increase and decrease. The bending and the radial mode of the piezoceramic are differently influenced by changing the length of the actuator. Therefore, for a specific frequency both modes superimpose. As a result the amplitudes of the excited $S_{0}$-mode are increased. An optimal energy efficient actuator design tries to superimpose both resonances.

The variation of the thickness of the actuator shows that the optimal Lamb wave excitation is not only dependent on the length of the actuator. For greater thicknesses the frequency of the first maxima of the symmetric mode is reduced. The amplitude of this maximum increases for a greater thickness. This numerical result agrees with the experimental findings and disagrees with "shear lag" predictions. The resonances of the actuator have a great impact on the excited Lamb wave mode. This effect covers the "shear lag" effect and is more dominant in influencing Lamb wave amplitudes than the adapted length proposed by Giurgiutiu (2008). The variation of the plate Young's modulus shows that the position of the first peak in the $\mathrm{S}_{0}$-mode spectrum is dominantly influenced by the piezoceramic source. The amplitudes, except for soft plates $\left(Y_{p l}<10^{11} \mathrm{~N} / \mathrm{m}^{2}\right)$, show only small variations.

In summary the design of an optimal shaped piezoceramic actuator (working in resonance) could be made for a fixed adhesive layer and a given plate material. Small changes in the adhesive layer, actuator geometry and plate stiffness do not dominantly change the behavior of the piezoceramic source.

\section{References}

Adam, C., Bouabdallah, S., Zarroug, M. and Maitournam, H. (2015), "Stable time step estimates for NURBS-based explicit dynamics”, Comput. Meth. Appl. Mech. Eng., 295(581-605), doi: http://dx.doi.org/10.1016/j.cma.2015.03.017.

Ahmad, Z.A.B. and Gabbert, U. (2012), "Simulation of Lamb wave reflections at plate edges using the semianalytical finite element method", Ultrasonics, 52(7), 815-820.

Ahmad, Z.A.B. (2011), "Numerical Simulations of Lamb waves in plates using a semi-analytical finite element method", Ph.D. thesis, Otto-von-Guericke-University of Magdeburg, Fortschritt-Berichte VDI Reihe 20, Nr. 437, Düsseldorf: VDI Verlag, ISBN:978-3-18-343720-7. 
Alleyne, D. and Cawley, P. (1991), "A two-dimensional Fourier transform method for the measurement of propagating multimode signals", J. Acoust. Soc. Am., 89(3), 1159-1168.

Bazilevs, Y. (2006), "Isogeometric analysis of turbulence and fluid-structure interaction", Ph.D. thesis, The University of Texas at Austin.

Boller, C., Chang, F.-K. and Fijino, Y. (2009), Encyclopedia of Structural Health Monitoring, John Wiley \& Sons, ISBN-10: 0470058226.

Cottrell, J.A., Hughes, T.J.R. and Bazilevs, Y. (2009), Isogeometric analysis: Toward integration of CAD and FEA, John Wiley \& Sons, ISBN-10: 0470748737.

Crawley, E.F. and de Luis, J. (1987), "Use of piezoelectric actuators as elements of intelligent structures", AIAA J., 25(10), 1373-1385.

de Boor, C. (1972), “On calculating with B-splines”, J. Approx. Theory, 6(1), 50-62.

Duczek, S. (2014), "Higher order finite elements and the fictitious domain concept for wave propagation analysis", Ph.D. thesis, Otto-von-Guericke-University of Magdeburg, Fortschritt-Berichte VDI Reihe 20, Nr. 458, Dusseldorf: VDI Verlag, ISBN: 978-3-18-345820-2.

Evans, J.A., Bazilevs, Y., Babuska, I. and Hughes, T.J.R. (2009), "n-Widths, sup-infs, and optimality ratios for the k-version of the isogeometric finite element method", Comput. Meth. Appl. Mech. Eng., 198(21), 1726-1741.

Giurgiutiu, V. (2008), Structural Health Monitoring with Piezoelectric Wafer Active Sensors, Academic Press (Elsevier), ISBN-13: 978-0-12-088760-6.

Ha, S. (2009), "Modeling Lamb wave propagation induced by adhesively bonded PZTs on thin plates", Ph.D. thesis, Stanford University, California, USA.

Ha, S. (2010), "Adhesive interface layer effects in PZT-induced Lamb wave propagation”, Smart Mater. Struct., 19(2), 025006.

Huang, C.-H., Lin, Y.-C. and Ma, C.-C. (2004), "Theoretical analysis and experimental measurement of resonant vibration of piezoceramic circular plates", IEEE Trans. Ultrasonic., Ferroelect., Freq. Control, 51(1), 12-24.

Hughes, T.J.R., Cottrell, J.A. and Bazilevs, Y. (2005), "Isogeometric analysis: CAD, finite elements, NURBS, exact geometry and mesh refinement", Comput. Meth. Appl. Mech. Eng., 194(39), 4135-4195.

Ikeda, T. (1996), Fundamentals of piezoelectricity, Oxford science publications, Oxford University Press, ISBN 9780198564607.

Kessler, S., Spearing, M. and Atallab, M. (2002), "In-situ damage detection of composites structures using Lamb wave methods", In Proceedings of the First European Workshop on Structural Health Monitoring, 10-12 July 2002 Paris France.

Marinkovic, D., Köppe, H. and Gabbert, U. (2006), "Numerically efficient finite element formulation for modeling active composite laminates", Mech. Adv. Mater. Struct., 13(5), 379-392.

Mohamed, R., Demer, D.L. and Masson, P. (2011), "A parametric study of piezoelectric thickness effect on the generation of fundamental Lamb modes", Hlth. Monit. Struct. Biol. Syst., Proceedings of SPIE Vol. 7984.

Mook, G., Pohl, J. and Michel, F. (2003), "Non-destructive characterization of smart CFRP structures", Smart Mater. Struct., 12(6), 997-1004.

PI Ceramic GmbH, Lederhose - GERMANY (2011), http://www.piceramic.com/index.php.

Piegl, L. and Tiller, W. (1995), The NURBS Book, Springer, ISBN: 3-540-55069-0.

Pohl, J., Willberg, C., Gabbert, U. and Mook, G. (2012), "Theoretical analysis and experimental determination of the dynamic behaviour of piezoceramic actuators for SHM", Exp. Mech., 51(4), 429-438.

Samal, M.K., Seshu, P., Parashar, S., von Wagner, U., Hagedorn, P., Dutta, B.K. and Kushwaha, H.S. (2005), "A finite element model for nonlinear behaviour of piezoceramics under weak electric fields", Finit. Element. Anal. Des., 41(15), 1464-1480.

Schmicker, D., Duczek, S., Liefold, S. and Gabbert, U. (2014), "Wave propagation analysis using high-order finite element methods: Spurious oscillations excited by internal element eigenfrequencies", Technische Mechanik, 34(2), 51-71.

Sirohi, J. and Chopra, I. (2000), "Fundamental understanding of piezoelectric strain sensors", J. Intellig. 
Mater. Syst. Struct., 11(4), 246-257.

Sohn, H. and Lee, S.J. (2010), "Lamb wave tuning curve calibration for surface-bonded piezoelectric Transducers", Smart Mater. Struct., 19(1), 015007.

$\mathrm{Su}, \mathrm{Z}$. and Ye, L. (2009), Identification of Damage Using Lamb Waves: From Fundamentals to Applications, Springer, ISBN: 978-1-84882-783-7.

Su, Z., Ye, L. and Lu, Y. (2006), "Guided Lamb waves for identification of damage in composite structures: A review", J. Sound Vib., 295(3), 753-780.

Willberg, C. (2013), "Development of a new isogeometric finite element and its application for Lamb wave based structural health", Ph.D. thesis, Otto-von-Guericke-University of Magdeburg, Fortschritt-Berichte VDI Reihe 20, Nr. 446, Dusseldorf: VDI Verlag, ISBN: 978-3-18-344620-9.

Willberg, C. and Gabbert, U. (2012), "Development of a three-dimensional piezoelectric isogeometric finite element for smart structure applications", Acta Mechanica, 223(8), 1837-1850.

Willberg, C., Vivar-Perez, J.M., Ahmad, Z. and Gabbert, U. (2009), "Simulation of piezoelectric induced Lamb wave motion in plates", In Proceedings of the 7th International Workshop on Structural Health Monitoring 2009: From System Integration to Autonomous Systems, 2299-2307.

Willberg, C., Duczek, S., Vivar-Perez, J.M., Schmicker, D. and Gabbert, U. (2012a), "Comparison of different higher order finite element schemes for the simulation of Lamb waves", Comput. Meth. Appl. Mech. Eng., 241, 246-261.

Willberg, C., Koch, S., Mook, G., Gabbert, U. and Pohl, J. (2012b), "Continuous mode conversion of Lamb waves in CFRP plates", Smart Mater. Struct., 21(7), 1-9.

Willberg, C., Duczek, S. and Gabbert, U. (2013), "Increasing the scanning range of Lamb wave based SHM systems by optimizing the actuator sensor design", CEAS Aeronaut. J., 4(1), 87-98.

Willberg, C., Duczek, S., Vivar-Perez, J.M. and Ahmad, Z.A.B. (2015), "Simulation methods for guided wave-based structural health monitoring: A review", Appl. Mech. Rev., 67(1), 010803.

Zienkiewicz, O.C. and Taylor, R. (2000), The Finite Element Method, Volume 1: Basis, ButterworthHeinemann, ISBN: 0750650494.

$C C$ 